\title{
Indirect interaction webs on tall goldenrod: community consequences of herbivore- induced phenotypes and genetic variation of plants
}

\section{$\operatorname{AUTHOR}(\mathrm{S})$ :}

Ohgushi, Takayuki; Ando, Yoshino; Utsumi, Shunsuke; Craig, Timothy P.

\section{CITATION:}

Ohgushi, Takayuki ...[et al]. Indirect interaction webs on tall goldenrod: community consequences of herbivore-induced phenotypes and genetic variation of plants. Journal of Plant Interactions 2011, 6(2-3): 147-150

\section{ISSUE DATE:}

2011-06

URL:

http://hdl.handle.net/2433/156521

\section{RIGHT:}

〔C 2011 Taylor \& Francis; 許諾条件により本文は2012-07-01に公開; この 論文は出版社版でありません。引用の際には出版社版をご確認ご利用 ください。; This is not the published version. Please cite only the published version. 
Indirect interaction webs on tall goldenrod: community consequences of herbivore-induced phenotypes and genetic variation of plants

\author{
Takayuki Ohgushi ${ }^{1}$, Yoshino Ando ${ }^{1}$, Shunsuke Utsumi ${ }^{1,2}$, and Timothy P. Craig ${ }^{3}$ \\ ${ }^{1}$ Center for Ecological Research, Kyoto University, Japan, ${ }^{2}$ Department of General \\ Systems Studies, University of Tokyo, Japan, ${ }^{3}$ Department of Biology, University of \\ Minnesota Duluth, USA
}

\begin{abstract}
Tall goldenrod, Solidago altissima, was introduced from North America 100 years ago, and it has become widely distributed all over Japan. We have been investigating plant-based indirect interaction webs on the tall goldenrod in Japan and the US. We found that arthropod communities on Japanese goldenrods are organized via plant- and ant-mediated indirect effects caused by the dominant aphid, Uroleucon nigrotuberculatum, which came from the US in early 1990's. The aphid also generated bottom-up cascading effects on temporally-separated communities. Our results highlight the importance of herbivore-induced plant phenotypes in determining the structure of indirect interaction webs. We review our ongoing research that focuses on the effect of plant genotypic variation on the formation of indirect interaction webs. Our findings of the genotypic effects on aphid population dynamics and herbivore community composition provide a more complete understanding of the community consequences of plant genotypic diversity, as well as herbivore-induced plant phenotypes.
\end{abstract}

\title{
Introduction
}

A major challenge for ecologists today is to understand how, and to what extent, interspecific interactions directly and indirectly govern the community structure and biodiversity in multitrophic systems (Duffy et al. 2007; Verhoef and Olff 2010). The focus on direct, trophic interactions in much of the research to date means that the potential significance of non-trophic, indirect, and facilitative interactions has been largely ignored in studies of interactions in multitrphic systems. However, these interactions are all very common and widespread in terrestrial systems. Incorporating non-trophic, indirect links into traditional food webs to create interaction webs (Ohgushi 2005) allows us to explore fully each species' impact on its community. Food webs 
provide an incomplete picture of the forces structuring arthropod communities because they ignore the indirect plant-mediated effects that are ubiquitous in a wide range of terrestrial plants (Ohgushi et al. 2007). To gain a more complete understanding of species interactions Ohgushi (2005) proposed the idea of an "indirect interaction web", which includes non-trophic, facilitative indirect links, representing the linkage of multiple plant-herbivore interactions. In particular, interaction linkages caused by herbivore-induced alterations in plant phenotypes have the potential to strongly influence the ecological communities by shaping the network of species interactions on terrestrial plants. Indirect interaction webs should be used to improve our understanding of the complexity of connections in an ecological community. Because they include critical information about non-trophic interactions, they provide a more complete understanding of community structure than can be provided by food webs alone.

Recently, there has been an increasing appreciation of the interplay of ecology and evolution in structuring communities (Johnson and Stinchcombe 2007). A new research field incorporating community genetics (Whitham et al. 2006), eco-evolutionary dynamics (Fussmann et al. 2007), and the geographic mosaic of coevolution (Thompson 2005) has rapidly developed that synthesizes the effects of evolution on ecological communities. The study of community genetics shows that genotype and genotypic variation of foundation species, such as dominant plant species, are of primary importance in structuring local communities. Thus, we need to incorporate the effects of herbivore-induced phenotypic plasticity and genotypic variation among plants into interaction networks to gain a more sophisticated understanding of community organization and biodiversity and how they are affected by species invasions.

In this review, we will briefly review our studies on arthropod indirect interaction webs on the tall goldenrod, to illustrate how non-trophic, indirect, and facilitative interactions play an important role in organizing plant-centered ecological communities. Then, we will discuss our ongoing research on herbivore community structure and population dynamics, with a focus on how plant genotypic variation and phenotypic plasticity in response to herbivores shape the structure of arthropod communities.

\section{Indirect interaction web on the tall goldenrod}

Tall goldenrod was introduced from the US approximately 100 years ago, and it has 
become widely distributed all over Japan. In the central and southern part of Japan the most common insect herbivores on the goldenrod are aphids, leafhoppers, and leaf-chewing geometrid caterpillars in spring and early summer, and grasshoppers and soft scales in autumn. The aphid Uroleucon nigrotuberculatum, which was introduced from North America in the early 1990s, is the most common insect herbivore from late June to late July. The native ant Formica japonica was frequently observed feeding on scattered aphid honeydew on adjacent leaves. We conducted an aphid removal experiment to examine the direct and indirect interactions imbedded in the indirect interaction web (Ando and Ohgushi 2008; Ando et al. in review; Figure 1). Aphid removal resulted in an eight times greater abundance of the leafhopper, Nephotettix cincticeps, and of the geometrid moth caterpillars, which was due to a decreased number of aphid-attending ants. However, aphid feeding changed neither nitrogen nor water content of the leaves during the early season. Interestingly, the impacts of aphid infestation were evidently transmitted to the soft scale, Parasaissetia nigra, and the grasshopper, Atractomorpha lata, in late September when the aphids were no longer present. The early-season aphid infestation increased leaf nitrogen and stimulated lateral shoot production in autumn. Aphid removal resulted in a four-fold increase of soft scales, probably because decreased phloem quality in aphid-exposed plants reduced colonization by the soft scales. As the soft scales are tended by F. japonica, the number of ants was significantly greater on aphid-free plants than on aphid-exposed plants. Conversely, feeding damage by grasshoppers on aphid-exposed plants was twice that on aphid-free plants. Grasshopper density increased on aphid-exposed plants because of increased leaf nitrogen and decreased impact of ants tending scale insects. Thus, aphids early in the season negatively affected soft scales by decreasing food quality, but positively influenced grasshoppers through increased leaf quality and decreased ants tending on the soft scales.

The goldenrod food web had five direct plant-insect interactions: feeding interactions consisting of aphids, leafhoppers, and geometrid leaf chewers in spring and early summer, and soft scales and grasshoppers in autumn. As early feeders are temporally separated from autumn feeders, there cannot be direct competition between them for food resources. In the indirect interaction web, the following indirect interactions were detected: the negative interaction between aphids and leafhoppers through ants tending aphids for honeydew, the negative interaction between aphids and 
leaf chewers through tending actions of ants early in the season, the negative interaction between aphids and soft scales through changes in sap quality, the positive interaction between aphids and grasshoppers through increased leaf nitrogen, and the negative interaction between soft scales and grasshoppers through tending ants for soft scale honeydew late in the season. In addition, five direct interactions were newly established: the positive interaction between aphids and ants, the negative interaction between ants and leafhoppers, the negative interaction between ants and leaf chewers in spring and early summer, the positive interaction between soft scales and ants, and the negative interaction between ants and grasshoppers in autumn. Thus, the indirect interaction web has eleven direct and four indirect interactions, including three positive interactions, while the food web approach encompassed only five negative, direct interactions. Our study of the indirect interaction web has revealed that indirect, nontrophic, and mutualistic interactions are 33,60 , and $12 \%$ of total interactions, respectively, in contrast with the lack of such interactions among these species in the food web.

We also found herbivore-initiated bottom-up cascading effects in the tall goldenrod (Ando et al. in review). The aphid removal experiment showed that the aphid indirectly influenced community properties of herbivores and predators in the early (aphid present) and late (aphid absent) season in a different way. Although the aphid did not influence the species richness of herbivores and predators, early-season aphid removal resulted in a $174 \%$ increase in abundance of herbivores and a $53 \%$ decrease in predators. Leafhopper and the geometrid moth caterpillars had 8 times greater abundance on aphid-free plants due to the decreased number of ants. The increased density of predators on aphid-infested plants was due to the increased number of ants. Ordination analysis showed that the community composition of both herbivores and predators significantly differed between aphid-infested and aphid-free plants. In late September when the aphids were no longer present, the species richness of herbivores and predators was unaffected by aphid colonization early in the season. On the other hand, the abundance of herbivores and predators was decreased by $22.9 \%$ and $46.6 \%$ on aphid-exposed plants. The density response of herbivores differed between the soft scale and the grasshopper. Aphid colonization in the early season resulted in 4.0-fold decrease of soft scales, probably because of a decline of phloem quality in aphid-exposed plants. As a result, number of ants tending soft scales also significantly decreased. Conversely, the grasshopper density on aphid-exposed plants was 3.3-times greater than on aphid-free 
plants. The increase in grasshopper density was due to increased leaf nitrogen and a decrease in the number of ants associated with scale insects. Hence, the community responses of herbivores and predators to the aphid removal significantly differed between the early and late seasons. It should be noted that the bottom-up cascading effects initiated by the early-season aphid attack apparently occurred in late season when aphids were no longer present because induced changes in plant traits occurred with a considerable delay. This indicates that herbivore-induced plant changes can initiate trophic cascades in a temporally-separated community. On the other hand, we did not detect such bottom-up cascades in the early season when aphids were present, because aphids did not change plant traits. Instead, the increased number of ants that were attracted to aphid honeydew had a significantly negative impact on the abundances of co-occurring herbivores.

\section{Plant genotypic effects on indirect interaction webs}

The advocates of the community genetics hypothesis argue that intraspecific genetic variations within a plant species, or in hybrid zones, can have important consequences for the community organization of herbivores and/or predators (Whitham et al. 2006). This is because plant genotypes vary in their resistance and susceptibility based on resource quality to multiple species of herbivores. Hence, different plant genotypes offer distinct niches for herbivorous arthropods to coexist. For example, genetically diverse goldenrod communities are more productive, and support greater arthropod species richness (Crutsinger et al. 2006) and pollinator abundance (Genung et al. 2010). Thus, plant genotypes have the potential to influence the arthropod indirect interaction webs on tall goldenrod as described above. To compare the direct and indirect interactions embedded in the arthropod communities on tall goldenrods in Japan and the US, we are conducting a series of field experiments using same goldenrod genotypes in the reciprocal transplant gardens in both countries, with focusing on how genotypic variation and/or herbivore-induced phenotypes of plants govern the formation of indirect interaction webs.

Here, we will briefly describe the two important results revealed by our preliminary field experiments that we will further examine in subsequent research. First, we conducted a common garden experiment to explore the effect of goldenrod genotypic diversity on the population dynamics of the aphid U. nigrotuberculatum in a common 
garden at the University of Minnesota Duluth (Utsumi et al. in review). To determine the impact of plant genotypic diversity on aphid population dynamics, we compared aphid densities in three treatments: single genotype plots, mixed genotype plots, and mixed genotype with cage plots. In the latter treatment plants were individually caged to prevent natural enemy attack and aphid movement among plants. We found that the synergistic effects on population growth increased aphid population size in the mixed genotype treatment, suggesting that the genetically diverse goldenrod population supports a greater abundance of the aphid metapopulation. We proposed two non-exclusive hypotheses to explain this pattern. One is that there is a source-sink relationship among genotypes with aphids moving from genotypes with high reproductive success to genotypes with low reproductive success. The alternative is that there is lower natural enemy mortality in the mixed genotype plots.

Second, we examined how variation in plant genotypes contributes to the structure in arthropod communities between Japan and North America. To accomplish this we transplanted ten genotypes each of tall goldenrods collected from Shiga in Japan and from Minnesota in the US to a common garden of Center for Ecological Research, Kyoto University and we have surveyed the arthropod communities since 2009. We found that the average aphid density was significantly higher on Shiga genotypes than on Minnesota genotypes. This suggests that the plant resistance against the aphid has a genetic basis, and that this genotype-dependent resistance may have declined since the introduction of tall goldenrods to Japan. In addition, the genetic similarity in plant genotypes is closely related to the similarity in herbivore community: a negative correlation between genetic distance among plant genotypes and similarity in the community composition of herbivores. One interesting pattern was that in the absence of the aphid no such effect of plant genotypes on community structure was found. This is probably because the aphid had a great impact on many of the other insect herbivores via ant- and plant-mediated indirect effects described above.

\section{Future directions}

Our results have confirmed that plant genotypes have the potential to influence the population dynamics and community structure of insect herbivores on tall goldenrods.

Along with clarifying the causal mechanisms responsible for population- and community-level consequences of plant genotypes on herbivores, we need to evaluate the 
relative importance of the plant phenotypic plasticity induced by herbivory and the genotypic variation among plants on the organization of indirect interaction webs on the introduced plant. In particular, we need to explore when and where these two factors independently, or in combination, influence the development of direct and indirect interaction networks, and biodiversity of plant-based ecological communities.

\section{Acknowledgements}

This study was supported by a Grant-in-Aid for Scientific Research of the Ministry of Education, Culture, Sports, Science, and Technology (A-15207003 and B-20370010) to T. Ohgushi, the Global COE program (A06) of Kyoto University, and the JSPS Core-to-Core Program.

\section{References}

Ando Y, Ohgushi T. 2008. Ant- and plant-mediated indirect effects induced by aphid colonization on herbivorous insects on tall goldenrod. Popul Ecol. 50:181-189.

Crutsinger GM, Collins MD, Fordyce JA, Gompert Z, Nice CC, Sanders NJ. 2006. Plant genotypic diversity predicts community structure and governs an ecosystem process. Science. 313:966-968.

Duffy JE, Cardinale BJ, France KE, McIntyre PB, Thébault E, Loreau M. 2007. The functional role of biodiversity in ecosystems: incorporating trophic complexity. Ecol Lett. 10:522-538.

Fussmann GF, Loreau M, Abrams PA. 2007. Eco-evolutionary dynamics of communities and ecosystems. Funct Ecol. 21:465-477.

Genung MA, Lessard J-P, Brown CB, Bunn WA, Cregger MA, Reynolds WN, Felker-Quinn E, Stevenson ML, Hartley AS, Crutsinger GM, Schweitzer JA, Bailey JK. 2010.

Non-additive effects of genotypic diversity increase flora abundance and abundace of floral visitors. PLoS ONE. 5:1-7.

Johnson MTJ, Stinchcombe JR. 2007. An emerging synthesis between community ecology and evolutionary biology. Trends Ecol Evol. 22:250-257.

Ohgushi T. 2005. Indirect interaction webs: herbivore-induced effects through trait change in plants. Annu Rev Ecol Evol System. 36:81-105. 
Ohgushi T. 2008. Herbivore-induced indirect interaction webs on terrestrial plants: the importance of non-trophic, indirect, and facilitative interactions. Entomol Exp et Appl. 128:217-229.

Ohgushi T, Craig TP, Price PW. 2007. Ecological Communities: Plant Mediation in Indirect Interaction Webs. Cambridge University Press, Cambridge, UK.

Thompson JN. 2005. The Geographic Mosaic of Coevolution. The University of Chicago Press, Chicago, USA.

Verhoef HA, Olff H. 2010. Trophic dynamics of communities. In: Verhoef, H. A. and P.J., M. (eds.), Community Ecology: Processes, Models, and Applications. Oxford University Press, pp. 25-36.

Whitham TG, Bailey JK, Schweitzer JA, Shuster SM, Bangert RK, Leroy CJ, Lonsdorf EV, Allan GJ, DiFazio SP, Potts BM, Fischer DG, Gehring CA, Lindroth RL, Marks JC, Hart SC, Wimp GM, Wooley SC. 2006. A framework for community and ecosystem genetics: from genes to ecosystems. Nat Rev Genet. 7:510-523.

\section{Legend of figures}

Figure 1. Top: Indirect interaction web of herbivorous insects on the goldenrod, Solidago altissima. Solid and dashed lines show direct and indirect links, respectively. Bottom: Number and types of interactions detected in food web and indirect interaction web. Modified from Ohgushi (2008). 
(

Ant


Goldenrod
Sep.-Oct.
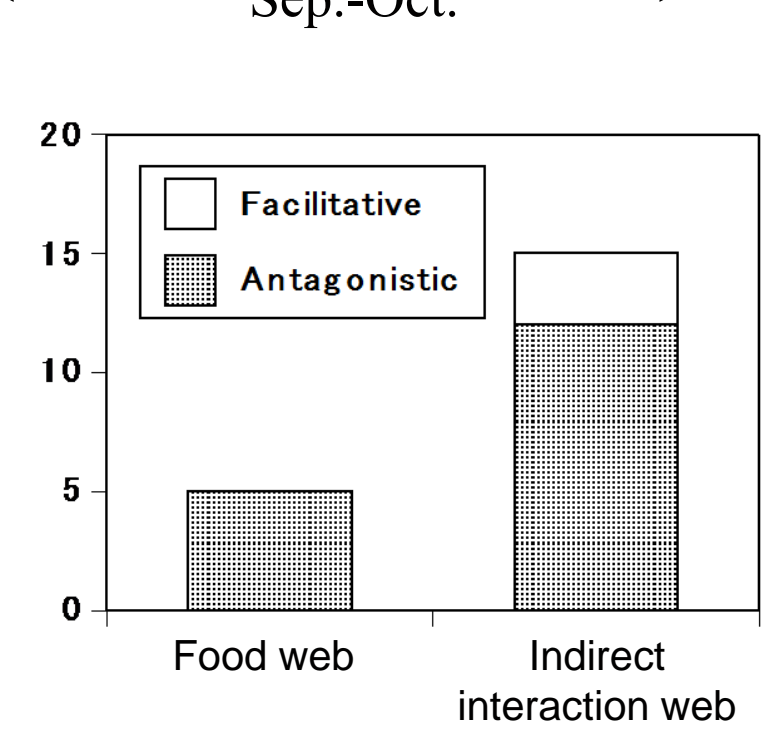
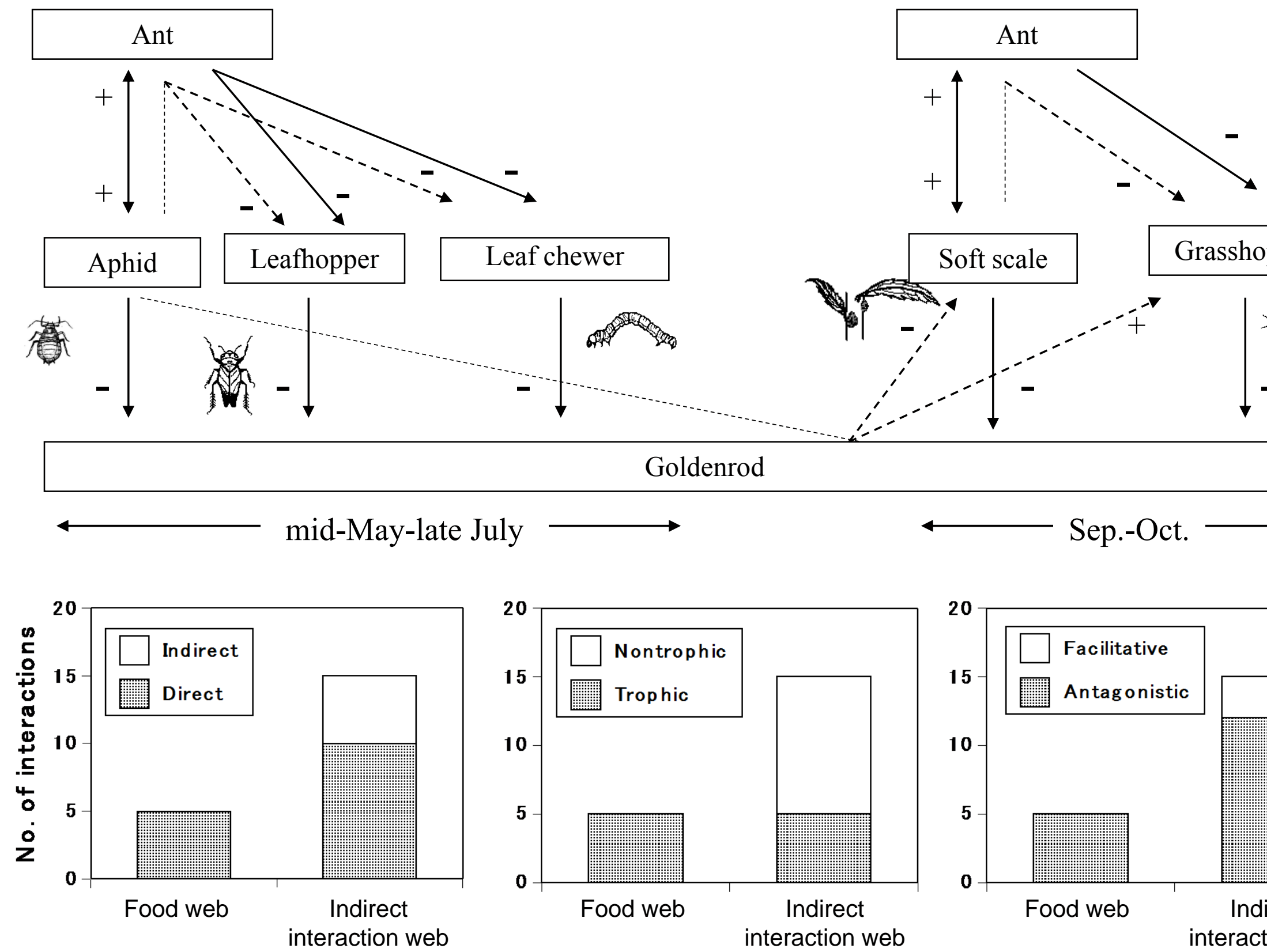

Grasshopper

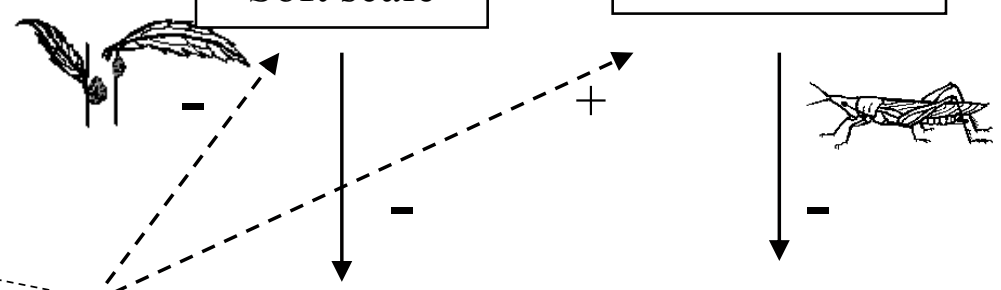

mid-May-late July 